\title{
Salvage helical tomotherapy for prostate cancer recurrence following definitive external beam radiotherapy: A case report
}

\author{
SANG HYUB LEE ${ }^{1}$, JINHONG JUNG $^{2}$ and SUNG-GOO CHANG ${ }^{1}$ \\ Departments of ${ }^{1}$ Urology and ${ }^{2}$ Radiation Oncology, \\ Kyung Hee University School of Medicine, Seoul 130-872, Republic of Korea
}

Received August 26, 2014; Accepted May 15, 2015

DOI: $10.3892 / \mathrm{ol} .2015 .3346$

\begin{abstract}
Salvage prostatectomy for recurrent prostate cancer after external beam radiotherapy (EBRT) has resulted in positive outcomes; however, it is technically challenging and is associated with a number of risks. When a patient exhibits recurrent prostate cancer following definitive EBRT and presents with comorbidities meaning that they cannot receive a prostatectomy, it is difficult to treat due to the numerous limitations of current salvage therapies. In the present study, two cases of salvage reirradiation using helical tomotherapy for locally recurrent prostate cancer following definitive EBRT are presented. The two patients received EBRT without severe complications. Local recurrence was confirmed by serum levels of prostate-specific antigen, repeat prostate biopsy, prostate magnetic resonance imaging and a bone scan. Salvage reirradiation using helical tomotherapy was performed and resulted in promising outcomes without any complications. In conclusion, helical tomotherapy can be a safe and effective salvage treatment modality for locally recurrent prostate cancer following definitive EBRT.
\end{abstract}

\section{Introduction}

External beam radiotherapy (EBRT) is one of the most commonly used primary treatment modalities for prostate cancer (1). However, 24-80\% of patients with high-risk prostate cancer experience biochemical relapse after definitive EBRT with or without androgen deprivation therapy (2-7). Current salvage therapies following primary EBRT include prostatectomy, cryosurgery, brachytherapy and high-intensity focused ultrasound (8). Although salvage prostatectomy has resulted in promising outcomes, it is generally recommended

Correspondence to: Professor Sung-Goo Chang, Department of Urology, Kyung Hee University School of Medicine, 23 Kyungheedae Road, Seoul 130-872, Republic of Korea

E-mail: sgchang@khu.ac.kr

Key words: intensity-modulated radiotherapy, local recurrence, prostate neoplasms, radiotherapy, salvage therapy that it be considered only in young patients without comorbidities and with low-risk prostate cancer. When a patient presents with recurrent prostate cancer following definitive EBRT and is deemed unfit for salvage prostatectomy, the most appropriate management strategy is difficult to determine due to the numerous limitations of current salvage therapies (9). Salvage re-irradiation for recurrent prostate cancer after primary EBRT has been limited due to toxicity to adjacent organs, particularly the rectum and bladder (10). Although, certain recent studies have reported the effectiveness and safety of re-irradiation with brachytherapy for locally relapsed prostate cancer, reports of re-irradiation using EBRT are scarce $(11,12)$. Thus, in the present study, two cases of locally relapsed prostate cancer following EBRT treated with re-irradiation using helical tomotherapy are discussed. The present study was approved by the ethics committee of Kyung Hee University Medical Center (Seoul, Republic of Korea).

\section{Case report}

Case 1. A 68-year-old male with a prostate-specific antigen (PSA) level of $15 \mathrm{ng} / \mathrm{ml}$ underwent a prostate biopsy and was diagnosed with localized prostate cancer with a Gleason score of 2+3 in October 2004. Prostate magnetic resonance imaging (MRI) and a bone scan showed organ confined prostate cancer. After a thorough consultation with the patient, he decided to undergo EBRT due to a history of cerebral infarction. A total dose of 72 Gy in 40 fractions was delivered to the prostate gland. After completion of definitive EBRT, he complained of mild urinary urgency and frequency, but these symptoms were controlled with anticholinergic agents. Seventeen months following completion of EBRT, the patient's PSA level decreased to $0.8 \mathrm{ng} / \mathrm{ml}$. However, in April 2007 (2 years and 3 months after EBRT) his PSA level increased to $1.37 \mathrm{ng} / \mathrm{ml}$, therefore, a repeat prostate biopsy was performed. Prostate cancer with a Gleason score of $3+3$ was diagnosed in one of eight cores. Prostate MRI and PET-CT revealed no distant metastasis or extra-prostatic tumor invasion. As the patient refused to undergo a salvage prostatectomy, intermittent androgen deprivation therapy was administered for 6 years. In 2013 (8 years and 6 months after the initial diagnosis), during the resting period the patient's PSA level reached $5.35 \mathrm{ng} / \mathrm{ml}$ within only 6 months. The patient underwent a further prostate biopsy and 2 out of 12 cores showed adenocarcinoma with a 

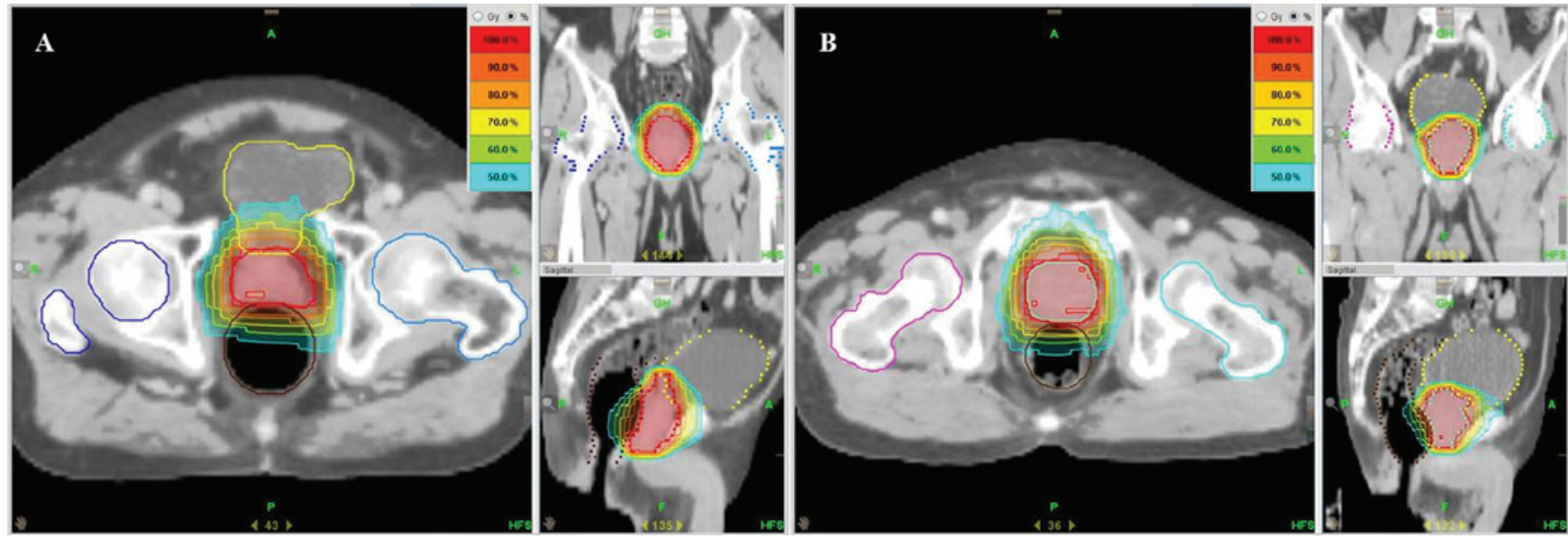

Figure 1. Isodose curves of the tomotherapy plans. (A) Case 1 and (B) case 2.

Gleason score of $4+4$. However, prostate MRI and a bone scan did not show periprostatic invasion or distant metastasis. After a thorough consultation, the patient decided to undergo tomotherapy. A total dose of 66 Gy in 30 fractions was administered using tomotherapy (Fig. 1A). Following tomotherapy, the patient's PSA level decreased to nadir and he did not experience any urologic problems. Furthermore, there have been no rectal complications thus far.

Case 2. A 71-year-old male with a PSA level of $8.7 \mathrm{ng} / \mathrm{ml}$ was diagnosed with Gleason score $3+3$ prostate cancer in November 2006. Extra-prostatic cancer was not detected by further imaging studies. The patient received EBRT as he refused a radical prostatectomy. A total dose of 77.4 Gy in 43 fractions was administered. Two years after EBRT, his PSA level decreased to $1.4 \mathrm{ng} / \mathrm{ml}$. However, it began to increase and in January 2010 (2 years and 11 months after EBRT) reached $2.6 \mathrm{ng} / \mathrm{ml}$. The patient underwent repeat prostate biopsy, which showed remaining cancer cells. A total dose of $69 \mathrm{~Gy}$ in 30 fractions was administered using tomotherapy (Fig. 1B). During the 4 years after tomotherapy, the PSA level did not increase from nadir and no rectal or urinary complications were reported.

\section{Discussion}

Salvage RT for cancer relapse after radical prostatectomy is an attractive option as the possible complications of RT associated with gastrointestinal (GI) or genitourinary (GU) toxicity are minimal, as described in a previous study (10). However, salvage reirradiation is considered a relative contraindication for recurrent prostate cancer after definitive EBRT (10). Recently Kishi et al (9) reported a case of salvage brachytherapy for recurrent prostate cancer after definitive EBRT. To minimize rectal toxicity, they used a bolus injection of native-type hyaluronic acid gel, which yielded promising oncologic and functional results. In another retrospective study of 37 patients who received salvage brachytherapy after definitive EBRT for prostate cancer, Aaronson et al (13) reported that brachytherapy provided prostate cancer control with an acceptable rate of complications. In their study, 10 patients experienced GI or GU complication. Functional outcomes and complications, especially GI and GU complications, following radiation therapy are graded from 1 to 4 (14). Only one patient experienced grade 3 rectal hemorrhage, while others experienced grade 2 complications that were managed conservatively. A similar study of 15 patients, who underwent brachytherapy for the treatment of recurrence after definitive EBRT, showed a biochemical relapse-free survival rate of $60.2 \% 3$ years after salvage brachytherapy and all acute adverse events were grade 1 or 2 (12). Furthermore, Ramey and Marshall (15) investigated 18 previous studies of salvage brachytherapy and concluded that brachytherapy is a reasonable salvage option for patients with local recurrence after primary EBRT for prostate cancer. Thus, according to previous studies of salvage brachytherapy, which described good oncologic outcomes with a tolerable rate of complications, salvage RT using brachytherapy is an acceptable treatment modality for the treatment of recurrent prostate cancer after definitive EBRT.

Helical tomotherapy, one of the newest conformal RT modalities, employs helical intensity-modulated RT in which a gantry 6-MV linear accelerator rotates continuously $360^{\circ}$ around the patient emitting tens of thousands of narrow beamlets providing an integrated megavoltage computed tomography unit that permits real-time verification of the patient's position during CT stimulation and treatment. Helical tomotherapy planning has numerous advantages, including a more conformal dose distribution and decreased radiation dose to normal structures. Low rates of GI and GU toxicity were observed in our previous study of 70 patients with localized prostate cancer treated with hypofractionated helical tomotherapy (16). In that study, all complications of the GI and GU tracts were grade 0,1 or 2, and either resolved spontaneously or could be managed with medication or simple procedures, such as argon plasma coagulation. In the present study, neither of the cases experienced complications greater than grade 3 . In case 1, analysis of dose volume histograms (DVHs) for the initial and salvage RT plans indicated that $<50 \%$ of the rectal volume received $>75 \mathrm{~Gy}$, with $<10 \%$ receiving $>120 \mathrm{~Gy}$, and $<5 \%$ receiving $>130 \mathrm{~Gy}$. The DVHs also showed that $<50 \%$ of the bladder volume received $>75 \mathrm{~Gy},<10 \%$ received $>100 \mathrm{~Gy}$, and $<5 \%$ received $>115 \mathrm{~Gy}$. In case 2 , analysis of DVHs for the initial and salvage RT plans 
showed that $<50 \%$ of the rectal volume received $>70 \mathrm{~Gy}$, with $<10 \%$ receiving $>110 \mathrm{~Gy}$, and $<5 \%$ receiving $>120 \mathrm{~Gy}$. The DVHs also showed that $<50 \%$ of the bladder volume received $>70 \mathrm{~Gy},<10 \%$ received $>110 \mathrm{~Gy}$, and $<5 \%$ received $>125 \mathrm{~Gy}$. Radiation doses to the rectum and bladder of these two patients were high considering the tolerance dose of these organs. However, doses delivered by helical tomotherapy to $50 \%$ of the rectal volume were only $8.8 \mathrm{~Gy}$ and $2.7 \mathrm{~Gy}$ for case 1 and case 2, respectively and the doses delivered to $50 \%$ of the bladder volume were $5.2 \mathrm{~Gy}$ and 2.2 Gy for case 1 and case 2, respectively. Furthermore, time intervals from the first and second EBRT were 8 years 4 months and 3 years 4 months, respectively, which should have been enough time to recover from mild complication following initial RT. To the best of our knowledge, there is only one study regarding reirradiation using EBRT. Vavassori et al (11) reported cases of reirradiation using CyberKnife ${ }^{\circledR}$ for locally recurrent prostate cancer after primary EBRT (median dose of $80 \mathrm{~Gy}$ ). A total dose of $30 \mathrm{~Gy}$ in 5 fractions was administered, and no patients experienced severe urinary or rectal toxicity. Therefore, if it is possible to use a high precision RT technique, salvage EBRT can be considered an appropriate treatment option for locally recurrent prostate cancer.

In conclusion, reirradiation was performed for prostate cancer using helical tomotherapy, which resulted in a promising outcome without urologic or rectal complications. Thus, it suggests that helical tomotherapy may be a safe and effective salvage treatment modality for locally recurrent prostate cancer following definitive EBRT.

\section{References}

1. Grimm P, Billiet I, Bostwick D, Dicker AP, Frank S Immerzeel J, Keyes M, Kupelian P, Lee WR, Machtens S, et al: Comparative analysis of prostate-specific antigen free survival outcomes for patients with low, intermediate and high risk prostate cancer treatment by radical therapy. Results from the Prostate Cancer Results Study Group. BJU Int 109: 22-29, 2012.

2. Roach M III, Bae K, Speight J, Wolkov HB, Rubin P, Lee RJ, Lawton C, Valicenti R, Grignon D and Pilepich MV: Short-term neoadjuvant androgen deprivation therapy and external-beam radiotherapy for locally advanced prostate cancer: Long-term results of RTOG 8610. J Clin Oncol 26: 585-591, 2008.

3. Bolla M, Collette L, Blank L, Warde P, Dubois JB, Mirimanoff RO, Storme G, Bernier J, Kuten A, Sternberg C, et al: Long-term results with immediate androgen suppression and external irradiation in patients with locally advanced prostate cancer (an EORTC study): A phase III randomised trial. Lancet 360: 103-106, 2002.
4. Horwitz EM,Bae K, Hanks GE, Porter A, Grignon DJ, Brereton HD, Venkatesan V, Lawton CA, Rosenthal SA, Sandler HM, et al: Ten-year follow-up of radiation therapy oncology group protocol 92-02: A phase III trial of the duration of elective androgen deprivation in locally advanced prostate cancer. J Clin Oncol 26: 2497-2504, 2008.

5. Pilepich MV, Winter K, Lawton CA, Krisch RE, Wolkov HB, Movsas B, Hug EB, Asbell SO and Grignon D: Androgen suppression adjuvant to definitive radiotherapy in prostate carcinoma-long-term results of phase III RTOG 85-31. Int J Radiat Oncol Biol Phys 61: 1285-1290, 2005.

6. Denham JW, Steigler A, Lamb DS, Joseph D, Turner S, Matthews J, Atkinson C, North J, Christie D, Spry NA, et al: Short-term neoadjuvant androgen deprivation and radiotherapy for locally advanced prostate cancer: 10 -year data from the TROG 96.01 randomised trial. Lancet Oncol 12: 451-459, 2011.

7. D'Amico AV, Manola J, Loffredo M, Renshaw AA, DellaCroce A and Kantoff PW: 6-month androgen suppression plus radiation therapy vs radiation therapy alone for patients with clinically localized prostate cancer: A randomized controlled trial. JAMA 292: 821-827, 2004.

8. Heidenreich A, Bastian PJ, Bellmunt J, Bolla M, Joniau S, van der Kwast T, Mason M, Matveev V, Wiegel T, Zattoni F and Mottet N; European Association of Urology: EAU guidelines on prostate cancer. Part II: Treatment of advanced, relapsing and castration-resistant prostate cancer. Eur Urol 65: 467-479, 2014.

9. Kishi K, Sato M, Shirai S, Sonomura T and Yamama R: Reirradiation of prostate cancer with rectum preservation: Eradicative high-dose-rate brachytherapy with natural type hyaluronate injection. Brachytherapy 11: 144-148, 2012.

10. Zelefsky MJ, Levin EJ, Hunt M, Yamada Y, Shippy AM, Jackson A and Amols HI: Incidence of late rectal and urinary toxicities after three-dimensional conformal radiotherapy and intensity-modulated radiotherapy for localized prostate cancer. Int J Radiat Oncol Biol Phys 70: 1124-1129, 2008.

11. Vavassori A, Jereczek-Fossa BA, Beltramo G, De Cicco L, Fariselli L, Bianchi LC, Possanzini M, Bergantin A, DeCobelli O and Orecchia R: Image-guided robotic radiosurgery as salvage therapy for locally recurrent prostate cancer after external beam irradiation: Retrospective feasibility study on six cases. Tumori 96: 71-75, 2010.

12. Shimbo M, Inoue K, Koike Y, Katano S and Kawashima K: Salvage I seed implantation for prostate cancer with postradiation local recurrence. Urol Int 90: 294-300, 2013.

13. Aaronson DS, Yamasaki I, Gottschalk A, Speight J, Hsu IC, Pickett B, Roach M III and Shinohara K: Salvage permanent perineal radioactive-seed implantation for treating recurrence of localized prostate adenocarcinoma after external beam radiotherapy. BJU Int 104: 600-604, 2009.

14. Budäus L, Bolla M, Bossi A, Cozzarini C, Crook J, Widmark A and Wiegel T: Functional outcomes and complications following radiation therapy for prostate cancer: A critical analysis of the literature. Eur Urol 61: 112-127, 2012.

15. Ramey SJ and Marshall DT: Re-irradiation for salvage of prostate cancer failures after primary radiotherapy. World J Urol 31: 1339-1345, 2013

16. Kong M, Hong SE and Chang SG: Hypofractionated helical tomotherapy ( $75 \mathrm{~Gy}$ at $2.5 \mathrm{~Gy}$ per fraction) for localized prostate cancer: long-term analysis of gastrointestinal and genitourinary toxicity. Onco Targets Ther 7: 553-566, 2014. 